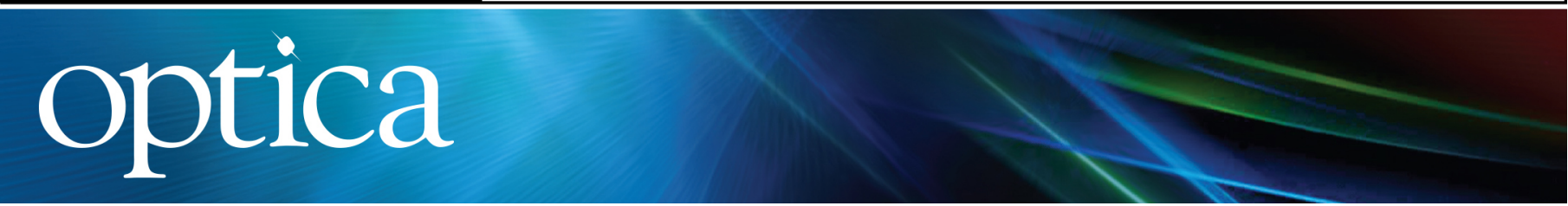

\title{
Quantum vampire: collapse-free action at a distance by the photon annihilation operator: supplementary material
}

\author{
Ilya A. Fedorovi1,2, Alexander E. Ulanovi1,3, Yury V. Kurochkin¹, ANd A. \\ I. LVOVSKY ${ }^{1,2,4}$, * \\ ${ }^{1}$ Russian Quantum Center, 100 Novaya Street, Skolkovo, Moscow 143025, Russia \\ ${ }^{2}$ P. N. Lebedev Physics Institute, Leninskiy prospect 53, Moscow 119991, Russia \\ ${ }^{3}$ Moscow Institute of Physics and Technology, 141700 Dolgoprudny, Russia \\ ${ }^{4}$ Institute for Quantum Science and Technology, University of Calgary, Calgary AB T2N 1N4, Canada \\ *Corresponding author: LVOV@ucalgary.ca
}

Published 29 January 2015

\begin{abstract}
This document provides supplementary information to "Quantum vampire: collapse-free action at a distance by the photon annihilation operator," http://dx.doi.org/10.1364/optica.2.000112. (C) 2015 Optical Society of America

http://dx.doi.org/10.1364/optica.2.000112.s001
\end{abstract}

The theoretical model used to describe our experiment is shown in Fig. S1. The heralded Fock state produced via parametric down-conversion is combined with the vacuum state $|0\rangle$ on beam splitter $\hat{B}_{1}$. One of the beam splitter outputs - the mode described by annihilation operator $\hat{a}_{1}-$ is subjected to photon annihilation using low-reflectivity beam splitter $\hat{B}_{2}$ and singlephoton detector $\mathrm{SPCM}_{3}$ in the reflected channel. Subsequently modes $\hat{a}_{1}$ and $\hat{a}_{2}$ are recombined on beam splitter $\hat{B}_{3}$. The output mode with constructive interference is subjected to homodyne detection after undergoing loss on an additional beam splitter $\hat{B}_{4}$.

The density matrix of the output state is calculated in the Fock basis using the matrix of the beam splitter operator, which can be found, for example, in Ref. [2]. Below, we present a more detailed analysis of the photon annihilation operation.

The action of beam splitter $\hat{B}_{2}$ can be modeled as evolution under Hamiltonian [1]

$$
\hat{H}=i \lambda \hat{a}_{1}\left(\hat{a}_{1}^{\prime}\right)^{\dagger}-i \lambda \hat{a}_{1}^{\dagger} \hat{a}_{1}^{\prime},
$$

where $\lambda$, assumed real, is related to the amplitude reflectivity $r$ of the beam splitter as $r=\sin (\zeta)$, with $\zeta=\lambda \tau / \hbar$ and $\tau$ being the fictitious interaction time. If $|\zeta| \ll 1$, the evolution under Hamiltonian (S1) can be approximated as

$$
\hat{B}_{2} \equiv e^{-i \hat{H} \tau / \hbar} \approx 1-i \hat{H} \tau / \hbar .
$$

Suppose the beam splitter input consists of state $|\psi\rangle$ in mode $\hat{a}_{1}$ and the vacuum state $|0\rangle$ in mode $\hat{a}_{1}^{\prime}$. The evolution operator, acting on this state, yields

$$
e^{-i \hat{H} \tau / \hbar}(|\psi\rangle|0\rangle) \approx|\psi\rangle|0\rangle+\zeta\left(\hat{a}_{1}|\psi\rangle\right)|1\rangle .
$$

Conditioning on single-photon detection in mode $\hat{a}_{1}^{\prime}$, we obtain state $\hat{a}_{1}|\psi\rangle$ in mode $\hat{a}_{1}$.

This approximation is valid as long as higher-order terms in Taylor decomposition Eq. (S2) can be neglected. These terms enter the output state with probabilities on a scale of $r^{2} n_{|\psi\rangle} \ll 1$, with $n_{|\psi\rangle}$ being the mean photon number in the target state. Fig. S2 shows the effect of neglecting higher-order terms on the expected output state.

After the beam splitter, mode $\hat{a}_{1}^{\prime}$ is subjected to measurement by non-discriminating single-photon detector $\mathrm{SPCM}_{3}$. This detector is descried by positive operator-valued measure [3]

$$
\begin{aligned}
& \hat{\Pi}_{\mathrm{off}}(\eta)=\sum_{n=0}^{\infty}\left(1-p_{\mathrm{bg}}\right)(1-\eta)^{n}|n\rangle\langle n| ; \\
& \hat{\Pi}_{\mathrm{on}}(\eta)=\sum_{n=0}^{\infty}\left[1-\left(1-p_{\mathrm{bg}}\right)(1-\eta)^{n}\right]|n\rangle\langle n|,
\end{aligned}
$$

where $\eta$ is the quantum efficiency of that detector and $p_{\mathrm{bg}}$ is the probability of a "background" click to occur in the absence of input photons. If the detector clicks, the state in mode $\hat{a}_{1}$ is projected onto

$$
S_{\hat{a}_{1}}(\hat{\rho})=\operatorname{Tr}_{\hat{a}_{1}^{\prime}}\left[\hat{\Pi}_{\text {on }} \hat{B}_{2}(\hat{\rho} \otimes|0\rangle\langle 0|) \hat{B}_{2}^{\dagger}\right],
$$

where $\hat{\rho}=|\psi\rangle\langle\psi|$ is the density matrix of the initial state in mode $\hat{a}_{1}$. 


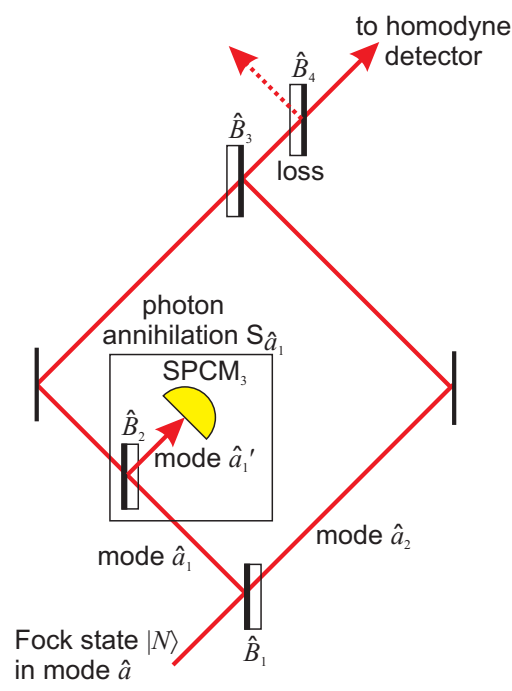

Fig. S1. Model of the experiment.
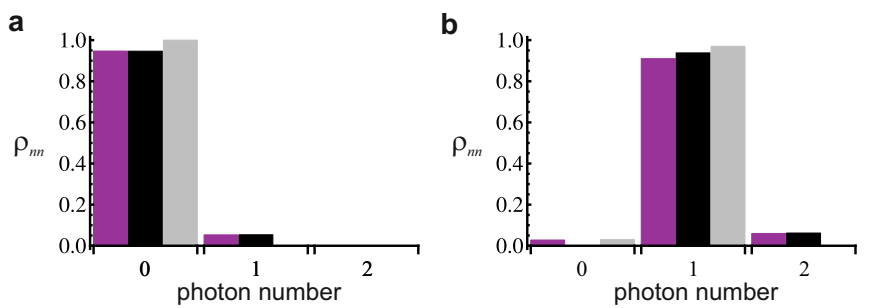

Fig. S2. Diagonal elements of the expected output state's density matrix in the Fock basis for one- (a) and two-photon (b) experiment. Purple rectangles: the approximation-free calculation according to Eq. (S5), same as in Fig. 3 of the main text. Black rectangles: a calculation using the first-order approximation (S3) of the beam splitter operator. Gray rectangles: calculation with no background clicks of the SPCM 3.

\section{REFERENCES}

1. I. Jex, S. Stenholm, A. Zeilinger, Opt. Comm. 117, 95-101 (1995).

2. U. Leonhardt, Measuring the Quantum state of Light (Cambridge University Press, Cambridge, 1997).

3. P. K. Kok and B. W. Lovett, Introduction to Optical Quantum Information Processing (Cambridge University Press, 2010). 